Pol. Con. (Edición núm. 5) Vol. 2, No 3 Marzo 2017

Revista científico-profesional en formato digital, perteneciente a la Casa Editora del Polo, de Manta, Ecuador. Posee una frecuencia de salida mensual e incluye la posibilidad de publicar números monográficos y especiales.

La Revista posee un diseño que asegura la eficiencia y rapidez en el proceso de evaluación de los trabajos, teniendo como premisa esencial el rigor científico, técnico y profesional. Todos los textos enviados son sometidos a una revisión por pares en la modalidad de doble ciego. Es una revista de acceso abierto (http://polodelconocimiento.com/ojs/index. php/es).

Se dirige a la comunidad científica nacional e internacional en el campo de las ciencias, haciendo confluir el quehacer investigativo y científico de las personas vinculadas con las siguientes áreas del saber: ciencias de la salud, ciencias sociales y políticas, ciencias de la educación, ciencias de la comunicación, ciencias técnicas y aplicadas, ciencias naturales, artes y letras, ciencias económicas y empresariales, ciencias matemáticas, ciencias de la computación y ciencias del deporte.

\section{EQUIPO EDITORIAL}

Director

Dr. Víctor R. Jama-Zambrano, Universidad Laica Eloy Alfaro de Manabí, Extensión Chone, Chone, Ecuador, Casa Editora del Polo (CASEDELPO), Manta, Ecuador

\section{Editor}

Abg. Néstor D. Suarez-Montes, Casa Editora del Polo (CASEDELPO), Manta, Ecuador

\section{Editor de Sección}

Mg. Walter D. Cobeña-Loor, Universidad Particular San Gregorio de Portoviejo, Portoviejo, Ecuador

\section{Editora Técnica}

Lic. Jessica M. Mero-Vélez, Casa Editora del Polo (CASEDELPO), Manta, Ecuador

\section{Editores Asociados}

Tglo. Alejandro David Plúa Argoti, Universidad Central del Ecuador, Quito, Ecuador

Mg. Danny E. Alcívar Vélez, Universidad Particular San Gregorio de Portoviejo, Portoviejo, Ecuador

\section{Editor Académico}

Mg. Ricardo Giniebra-Urra, Universidad de la Habana, La Habana, Cuba

\section{Otros miembros del Consejo Editorial}

Dra. Maritza Berenguer- Gouarnaluses, Universidad Santiago de Cuba, Santiago de Cuba, Cuba

Mg. José G. Burgos-Briones, Colegio Nacional Manta, Manta, Ecuador

Dr. Oswaldo Zambrano-Quinde, Universidad Laica Eloy Alfaro de Manabí, Ecuador, Manta, Ecuador

Segundo N. Castillo-Cabeza, Universidad Luis Vargas Torres de Esmeraldas, Esmeraldas, Ecuador

Mg. Juan C. Mera-Cedeño, Universidad Particular San Gregorio de Portoviejo, Portoviejo, Ecuador

Mg. Lenin A. Párraga-Zambrano, Universidad Laica Eloy Alfaro de Manabí, Extensión Chone, Chone, Ecuador

\section{Comité Científico}

$\mathrm{PhD}$ José María Lalama-Aguirre, Universidad Central del Ecuador, Quito, Ecuador

Dr. Gustavo J. Machado-Macellaro, Universidad de la República, Uruguay

Dr. Adrián Cuevas Jiménez, Universidad Nacional Autónoma de México, FES IZTACALA, México

Mg. Karelín López Sánchez, Universidad de La Habana, La Habana, Cuba

\section{Equipo técnico}

Tec. Juan A. Berenguer-Gouarnaluses, Universidad de Ciencias Médicas, Santiago de Cuba, Cuba

Lic. María A. Mero Vélez, Casa Editora del Polo, (CASEDELPO), Manta, Ecuador 